CARPATHIAN J. MATH.

Volume 37 (2021), No. 3,

Pages 489 - 495
Online version at https : //www. carpathian. cunbm. utcluj.ro/

Print Edition: ISSN 1584 - 2851; Online Edition: ISSN 1843 - 4401

DOI: https://doi.org/10.37193/CJM.2021.03.11

Dedicated to Professor Jitsuro Sugie on the occasion of his $65^{\text {th }}$ birthday

\title{
Instability of second-order nonhomogeneous linear difference equations with real-valued coefficients
}

\author{
MASAKAZU ONITSUKA
}

\begin{abstract}
In J. Comput. Anal. Appl. (2020), pp. 152-165, the author dealt with Hyers-Ulam stability of the second-order linear difference equation $\Delta_{h}^{2} x(t)+\alpha \Delta_{h} x(t)+\beta x(t)=f(t)$ on $h \mathbb{Z}$, where $\Delta_{h} x(t)=$ $(x(t+h)-x(t)) / h$ and $h \mathbb{Z}=\{h k \mid k \in \mathbb{Z}\}$ for the step size $h>0 ; \alpha$ and $\beta$ are real numbers; $f(t)$ is a real-valued function on $h \mathbb{Z}$. The purpose of this paper is to clarify that the second-order linear difference equation has no Hyers-Ulam stability when the step size $h>0$ and the coefficients $\alpha$ and $\beta$ satisfy suitable conditions. Finally, a necessary and sufficient condition for Hyers-Ulam stability is obtained.
\end{abstract}

\section{INTRODUCTION}

In 1940, Ulam [22] posed one of the stability in functional equations. This stability will later be called Ulam stability or Hyers-Ulam stability. The study of Hyers-Ulam stability has evolved in the field of functional equations, and its concept has been extended to the field of differential equations, recurrence and dynamic equations on time scales (see $[1,2,3,4,5,6,7,8,9,10,11,12,13,14,15,16,17,18,19,20,21,23])$.

Recently, the author [16] studied Hyers-Ulam stability of the second-order nonhomogeneous linear difference equation

$$
\Delta_{h}^{2} x(t)+\alpha \Delta_{h} x(t)+\beta x(t)=f(t)
$$

on $h \mathbb{Z}$, where

$$
\Delta_{h} x(t)=\frac{x(t+h)-x(t)}{h} \quad \text { and } \quad h \mathbb{Z}=\{h k \mid k \in \mathbb{Z}\}
$$

for the constant step size $h>0 ; \alpha$ and $\beta$ are real numbers; $f(t)$ is a real-valued function on $h \mathbb{Z}$. We say that (1.1) has "Hyers-Ulam stability" on $h \mathbb{Z}$ if there exists a constant $K>0$ with the following property: Let $\varepsilon>0$ be a given arbitrary constant. If a function $\phi: h \mathbb{Z} \rightarrow \mathbb{R}$ satisfies $\left|\Delta_{h}^{2} \phi(t)+\alpha \Delta_{h} \phi(t)+\beta \phi(t)-f(t)\right| \leq \varepsilon$ for all $t \in h \mathbb{Z}$, then there exists a solution $x: h \mathbb{Z} \rightarrow \mathbb{R}$ of (1.1) such that $|\phi(t)-x(t)| \leq K \varepsilon$ for all $t \in h \mathbb{Z}$. We call such $K$ a "HUS constant" for (1.1) on $h \mathbb{Z}$. In addition, we call the minimum of HUS constants for (1.1) on $h \mathbb{Z}$ the "best HUS constant". In [16], the author presented the following result.

Theorem 1.1 (See [16, Theorem 2.1]). Suppose that the quadratic equation

$$
\lambda^{2}+\alpha \lambda+\beta=0
$$

has real roots $\lambda_{1}$ and $\lambda_{2}$ with $\lambda_{i} \neq-2 / h,-1 / h$ and 0 for $i \in\{1,2\}$. Then (1.1) has Hyers-Ulam stability with an HUS constant $K\left(\lambda_{1}, h\right) K\left(\lambda_{2}, h\right)$ on $h \mathbb{Z}$, where $K(\lambda, h)$ is defined by

$$
K(\lambda, h)=\frac{h}{|1-| \lambda h+1||}
$$

Received: 15.05.2020. In revised form: 30.05.2021. Accepted: 07.06.2021

2010 Mathematics Subject Classification. 39A30, 39B82, 39A06.

Key words and phrases. Hyers-Ulam stability, linear difference equation, constant step size. 
Define the set $S$ by

$$
S:=\left\{(\alpha, \beta) \in \mathbb{R}^{2} \mid \beta>\frac{\alpha^{2}}{4}, \beta \neq \frac{\alpha}{h}\right\} .
$$

Recently, the following result was obtained by Anderson and Onitsuka [4].

Theorem 1.2 (See [4, Theorem 3.3]). If $(\alpha, \beta) \in S$, then (1.1) has Hyers-Ulam stability with an HUS constant

$$
K\left(\frac{-\alpha+\sqrt{\alpha^{2}-4 \beta}}{2}, h\right) K\left(\frac{-\alpha-\sqrt{\alpha^{2}-4 \beta}}{2}, h\right)=\frac{1}{\left(\frac{1}{h}-\sqrt{\beta-\frac{\alpha}{h}+\frac{1}{h^{2}}}\right)^{2}}
$$

on $h \mathbb{Z}$, where $K(\lambda, h)$ is the constant given by (1.3) for $\lambda \in \mathbb{C}$.

Remark 1.1. It is known that $K(\lambda, h)$ is the best HUS constant for the first-order linear difference equation $\Delta_{h} x(t)-\lambda x(t)=0$ on $h \mathbb{Z}$ (see, $\left.[4,14,15]\right)$.

Remark 1.2. Under the assumption that $1-\alpha h+\beta h^{2} \neq 0$, we see that (1.2) is the characteristic equation for the second-order homogeneous linear difference equation

$$
\Delta_{h}^{2} x(t)+\alpha \Delta_{h} x(t)+\beta x(t)=0
$$

on $h \mathbb{Z}$, since $(\lambda h+1)^{t / h}$ is a solution of this equation on $h \mathbb{Z}$ if and only if (1.2) holds. If $1-\alpha h+\beta h^{2}=0$ then we no longer have a second-order difference equation, and we have $\lambda=-1 / h$. Conversely, $\lambda=-1 / h$ implies $1-\alpha h+\beta h^{2}=0$. For this reason, the assumption $\lambda_{i} \neq-1 / h$ for $i \in\{1,2\}$ in Theorem 1.1 is naturally assumed. Moreover, $(\alpha, \beta) \in S$ implies $\lambda_{i} \neq-1 / h$ for $i \in\{1,2\}$ in Theorem 1.2.

The following result naturally established from the Theorems 1.1 and 1.2.

Theorem 1.3. Let $\lambda_{1}$ and $\lambda_{2}$ be the complex roots of (1.2) with $\lambda_{i} \neq-1 / h$ for $i \in\{1,2\}$. If $\lambda_{i} \neq 0$ and $\lambda_{i} \neq-2 / h$ for $i \in\{1,2\}$, then (1.1) has Hyers-Ulam stability with an HUS constant $K\left(\lambda_{1}, h\right) K\left(\lambda_{2}, h\right)$ on $h \mathbb{Z}$, where $K(\lambda, h)$ is the constant given by (1.3) for $\lambda \in \mathbb{C}$.

An important question now arises. If there exists $j \in\{1,2\}$ such that $\lambda_{j}=0$ or $\lambda_{j}=$ $-2 / h$, does (1.1) have Hyers-Ulam stability? The main purpose of this study is to answer this question.

In the next section, we will give two lemmas that describes some calculations used for the proof of the main theorem. In section 3, we will present the main theorem and its proof. In section 4 , we will derive two theorems that can be judged by the real-valued coefficients $\alpha$ and $\beta$. Finally, in section 5, we give conclusions.

\section{LEMMAS}

In this section, we will give some simple lemmas. Let $\Phi(t)$ be an antidifference of $\phi(t)$ on $h \mathbb{Z}$, that is, $\Delta_{h} \Phi(t)=\phi(t)$ holds on $h \mathbb{Z}$. For any real constant $C$, we denote $\Phi(t)+C$ by $\Delta_{h}^{-1} \phi(t)$. The first lemma was given in [16].

Lemma 2.1 (See [16, Lemma 2.1]). Suppose that (1.2) has real roots $\lambda_{1}$ and $\lambda_{2}$ with $\lambda_{i} \neq-1 / h$ for $i \in\{1,2\}$. Define

$$
F(t)=\Delta_{h}^{-1} f(t)\left(\lambda_{2} h+1\right)^{-\frac{t+h}{h}}
$$

and

$$
Y\left(t ; \lambda_{1}, \lambda_{2}\right)=\left\{\Delta_{h}^{-1} F(t)\left(\lambda_{2} h+1\right)^{\frac{t}{h}}\left(\lambda_{1} h+1\right)^{-\frac{t+h}{h}}\right\}\left(\lambda_{1} h+1\right)^{\frac{t}{h}}
$$

for $t \in h \mathbb{Z}$. Then $Y\left(t ; \lambda_{1}, \lambda_{2}\right)$ is a solution of (1.1). 
Lemma 2.2. The following equalities hold:

$$
\begin{gathered}
\Delta_{h}(-1)^{\frac{t}{h}}=-\frac{2}{h}(-1)^{\frac{t}{h}} \\
\Delta_{h} t(-1)^{\frac{t}{h}}=-\frac{2}{h} t(-1)^{\frac{t}{h}}-(-1)^{\frac{t}{h}} \\
\Delta_{h} t^{2}(-1)^{\frac{t}{h}}=-\frac{2}{h} t^{2}(-1)^{\frac{t}{h}}-2 t(-1)^{\frac{t}{h}}-h(-1)^{\frac{t}{h}} .
\end{gathered}
$$

Proof. From the definition of $\Delta_{h}$, we have

$$
\begin{gathered}
\Delta_{h}(-1)^{\frac{t}{h}}=\frac{1}{h}\left\{(-1)^{\frac{t+h}{h}}-(-1)^{\frac{t}{h}}\right\}=-\frac{2}{h}(-1)^{\frac{t}{h}} \\
\Delta_{h} t(-1)^{\frac{t}{h}}=\frac{1}{h}\left\{(t+h)(-1)^{\frac{t+h}{h}}-t(-1)^{\frac{t}{h}}\right\}=\frac{1}{h}(2 t+h)(-1)^{\frac{t+h}{h}}=-\frac{2}{h} t(-1)^{\frac{t}{h}}-(-1)^{\frac{t}{h}}
\end{gathered}
$$

and

$$
\begin{aligned}
\Delta_{h} t^{2}(-1)^{\frac{t}{h}} & =\frac{1}{h}\left\{(t+h)^{2}(-1)^{\frac{t+h}{h}}-t^{2}(-1)^{\frac{t}{h}}\right\}=\frac{1}{h}\left(2 t^{2}+2 h t+h^{2}\right)(-1)^{\frac{t+h}{h}} \\
& =-\frac{2}{h} t^{2}(-1)^{\frac{t}{h}}-2 t(-1)^{\frac{t}{h}}-h(-1)^{\frac{t}{h}}
\end{aligned}
$$

for all $t \in h \mathbb{Z}$.

\section{INSTABILITY}

The answer to the question given in the first section is as follows.

Theorem 3.1. Let $\lambda_{1}$ and $\lambda_{2}$ be the complex roots of (1.2) with $\lambda_{i} \neq-1 / h$ for $i \in\{1,2\}$. If there exists $j \in\{1,2\}$ such that $\lambda_{j}=0$ or $\lambda_{j}=-2 / h$, then (1.1) does not have Hyers-Ulam stability on $h \mathbb{Z}$.

Proof. Let $\varepsilon>0$. To prove instability, we have only to show that for a given $y(t)$ such that

$$
\left|\Delta_{h}^{2} y(t)+\alpha \Delta_{h} y(t)+\beta y(t)-f(t)\right| \leq \varepsilon
$$

holds on $h \mathbb{Z}$, any solution $x(t)$ of (1.1) satisfies $|y(t)-x(t)| \rightarrow \infty$ as $t \rightarrow \infty$. The proof of Theorem 3.1 is divided into four cases: (a) $\lambda_{1}=\lambda_{2}=0$; (b) $\lambda_{1} \neq \lambda_{2}=0$; (c) $\lambda_{1} \neq \lambda_{2}=$ $-2 / h ;$ (d) $\lambda_{1}=\lambda_{2}=-2 / h$.

First, we prove the case (a) $\lambda_{1}=\lambda_{2}=0$. In this case, we can assume that $\alpha=\beta=0$. Let

for all $t \in h \mathbb{Z}$. Since

$$
y_{1}(t)=\frac{\varepsilon t^{2}}{2}+\Delta_{h}^{-2} f(t)
$$

$$
\Delta_{h} y_{1}(t)=\frac{\varepsilon}{2 h}\left\{(t+h)^{2}-t^{2}\right\}+\Delta_{h}^{-1} f(t)=\varepsilon t+\frac{h}{2}+\Delta_{h}^{-1} f(t)
$$

holds, we see that $y_{1}(t)$ is a solution of the equation

$$
\Delta_{h}^{2} y_{1}(t)-f(t)=\varepsilon
$$

on $h \mathbb{Z}$. Hence, $\left|\Delta_{h}^{2} y_{1}(t)-f(t)\right|=\varepsilon$ is satisfied on $h \mathbb{Z}$. It is clear that

$$
x_{1}(t)=c_{1} t+c_{2}+\Delta_{h}^{-2} f(t)
$$

is any solution of (1.1) with $\alpha=\beta=0$, where $c_{1}$ and $c_{2}$ are arbitrary constants. Thus, we get $\lim _{t \rightarrow \infty}\left|y_{1}(t)-x_{1}(t)\right|=\infty$ for any $c_{1}$ and $c_{2}$.

Next, we consider the case (b) $\lambda_{1} \neq \lambda_{2}=0$. That is, $\alpha=-\lambda_{1}$ and $\beta=0$. Let

$$
y_{2}(t)=-\frac{\varepsilon t}{\lambda_{1}}+Y\left(t ; \lambda_{1}, 0\right)
$$


for all $t \in h \mathbb{Z}$, where $Y$ is the function given by (2.4). From Lemma 2.1, $Y\left(t ; \lambda_{1}, 0\right)$ is a solution of (1.1) with $\alpha=-\lambda_{1}$ and $\beta=0$. Using this fact and

it follows that

$$
\Delta_{h} \frac{\varepsilon t}{\lambda_{1}}=\frac{\varepsilon}{\lambda_{1}} \quad \text { and } \quad \Delta_{h}^{2} \frac{\varepsilon t}{\lambda_{1}}=0,
$$

$$
\begin{aligned}
\Delta_{h}^{2} y_{2}(t)-\lambda_{1} \Delta_{h} y_{2}(t)-f(t) & =\Delta_{h}^{2}\left(-\frac{\varepsilon t}{\lambda_{1}}+Y\left(t ; \lambda_{1}, 0\right)\right)-\lambda_{1} \Delta_{h}\left(-\frac{\varepsilon t}{\lambda_{1}}+Y\left(t ; \lambda_{1}, 0\right)\right)-f(t) \\
& =\Delta_{h}^{2} Y\left(t ; \lambda_{1}, 0\right)-\lambda_{1} \Delta_{h} Y\left(t ; \lambda_{1}, 0\right)-f(t)+\varepsilon=\varepsilon
\end{aligned}
$$

for all $t \in h \mathbb{Z}$. That is, $y_{2}(t)$ is a solution of the equation

$$
\Delta_{h} y_{2}^{2}(t)-\lambda_{1} \Delta_{h} y_{2}(t)-f(t)=\varepsilon
$$

on $h \mathbb{Z}$. This implies that $\left|\Delta_{h} y_{2}^{2}(t)-\lambda_{1} \Delta_{h} y_{2}(t)-f(t)\right|=\varepsilon$ is satisfied on $h \mathbb{Z}$. It is clear that

$$
x_{2}(t)=c_{1}\left(\lambda_{1} h+1\right)^{\frac{t}{h}}+c_{2}+Y\left(t ; \lambda_{1}, 0\right)
$$

is any solution of (1.1) with $\alpha=-\lambda_{1}$ and $\beta=0$, where $c_{1}$ and $c_{2}$ are arbitrary constants. Hence, we get $\lim _{t \rightarrow \infty}\left|y_{2}(t)-x_{2}(t)\right|=\infty$ for any $c_{1}$ and $c_{2}$. Let

We now consider the case (c) $\lambda_{1} \neq \lambda_{2}=-2 / h$. That is, $\alpha=-\lambda_{1}+2 / h$ and $\beta=-2 \lambda_{1} / h$.

$$
y_{3}(t)=\frac{\varepsilon t(-1)^{\frac{t}{h}}}{\lambda_{1}+2 / h}+Y\left(t ; \lambda_{1},-2 / h\right)
$$

for all $t \in h \mathbb{Z}$, where $Y$ is the function given by (2.4). From Lemma $2.1, Y\left(t ; \lambda_{1},-2 / h\right)$ is a solution of (1.1) with $\alpha=-\lambda_{1}+2 / h$ and $\beta=-2 \lambda_{1} / h$. By Lemma 2.2, we have

$$
\begin{aligned}
\Delta_{h}\left(y_{3}(t)-Y\left(t ; \lambda_{1},-2 / h\right)\right) & =\frac{\varepsilon}{\lambda_{1}+2 / h}\left\{-\frac{2}{h} t(-1)^{\frac{t}{h}}-(-1)^{\frac{t}{h}}\right\} \\
& =-\frac{2}{h}\left(y_{3}(t)-Y\left(t ; \lambda_{1},-2 / h\right)\right)-\frac{\varepsilon(-1)^{\frac{t}{h}}}{\lambda_{1}+2 / h}
\end{aligned}
$$

for all $t \in h \mathbb{Z}$. That is

$$
\Delta_{h} y_{3}(t)+\frac{2}{h} y_{3}(t)=\Delta_{h} Y\left(t ; \lambda_{1},-2 / h\right)+\frac{2}{h} Y\left(t ; \lambda_{1},-2 / h\right)-\frac{\varepsilon(-1)^{\frac{t}{h}}}{\lambda_{1}+2 / h}
$$

holds for all $t \in h \mathbb{Z}$. Using this equality and Lemma 2.2, we have

$$
\begin{aligned}
\Delta_{h}^{2} y_{3}(t) & +\left(-\lambda_{1}+\frac{2}{h}\right) \Delta_{h} y_{3}(t)-\frac{2 \lambda_{1}}{h} y_{3}(t)-f(t) \\
& =\Delta_{h}\left(\Delta_{h} y_{3}(t)+\frac{2}{h} y_{3}(t)\right)-\lambda_{1}\left(\Delta_{h} y_{3}(t)+\frac{2}{h} y_{3}(t)\right)-f(t) \\
& =\Delta_{h}\left(-\frac{\varepsilon(-1)^{\frac{t}{h}}}{\lambda_{1}+2 / h}\right)-\lambda_{1}\left(-\frac{\varepsilon(-1)^{\frac{t}{h}}}{\lambda_{1}+2 / h}\right) \\
& =\frac{2 \varepsilon(-1)^{\frac{t}{h}}}{h\left(\lambda_{1}+2 / h\right)}+\frac{\lambda_{1} \varepsilon(-1)^{\frac{t}{h}}}{\lambda_{1}+2 / h}=\varepsilon(-1)^{\frac{t}{h}}
\end{aligned}
$$

for all $t \in h \mathbb{Z}$. This implies that

$$
\left|\Delta_{h}^{2} y_{3}(t)+\left(-\lambda_{1}+\frac{2}{h}\right) \Delta_{h} y_{3}(t)-\frac{2 \lambda_{1}}{h} y_{3}(t)-f(t)\right|=\varepsilon
$$

holds on $h \mathbb{Z}$. It is easy to check that

$$
x_{3}(t)=c_{1}\left(\lambda_{1} h+1\right)^{\frac{t}{h}}+c_{2}(-1)^{\frac{t}{h}}+Y\left(t ; \lambda_{1},-2 / h\right)
$$


is any solution of (1.1) with $\alpha=-\lambda_{1}+2 / h$ and $\beta=-2 \lambda_{1} / h$, where $c_{1}$ and $c_{2}$ are arbitrary constants. Hence, we obtain $\lim _{t \rightarrow \infty}\left|y_{3}(t)-x_{3}(t)\right|=\infty$ for any $c_{1}$ and $c_{2}$.

Finally we prove the case (d) $\lambda_{1}=\lambda_{2}=-2 / h$. Namely, $\alpha=4 / h$ and $\beta=4 / h^{2}$. Let

$$
y_{4}(t)=\frac{\varepsilon t^{2}(-1)^{\frac{t}{h}}}{2}+Y(t ;-2 / h,-2 / h)
$$

for all $t \in h \mathbb{Z}$, where $Y$ is the function given by (2.4). From Lemma 2.1, $Y(t ;-2 / h,-2 / h)$ is a solution of (1.1) with $\alpha=4 / h$ and $\beta=4 / h^{2}$. By Lemma 2.2, we have

$$
\begin{aligned}
\Delta_{h}\left(y_{4}(t)-Y(t ;-2 / h,-2 / h)\right) & =\frac{\varepsilon}{2}\left\{-\frac{2}{h} t^{2}(-1)^{\frac{t}{h}}-2 t(-1)^{\frac{t}{h}}-h(-1)^{\frac{t}{h}}\right\} \\
& =-\frac{2}{h}\left(y_{4}(t)-Y(t ;-2 / h,-2 / h)\right)-\varepsilon t(-1)^{\frac{t}{h}}-\frac{h \varepsilon}{2}(-1)^{\frac{t}{h}}
\end{aligned}
$$

for all $t \in h \mathbb{Z}$. That is,

$$
\Delta_{h} y_{4}(t)+\frac{2}{h} y_{4}(t)=\Delta_{h} Y(t ;-2 / h,-2 / h)+\frac{2}{h} Y(t ;-2 / h,-2 / h)-\varepsilon t(-1)^{\frac{t}{h}}-\frac{h \varepsilon}{2}(-1)^{\frac{t}{h}}
$$

holds for all $t \in h \mathbb{Z}$. Using this equality and Lemma 2.2, we have

$$
\begin{aligned}
\Delta_{h}^{2} y_{4}(t) & +\frac{4}{h} \Delta_{h} y_{4}(t)+\frac{4}{h^{2}} y_{4}(t)-f(t) \\
& =\Delta_{h}\left(\Delta_{h} y_{4}(t)+\frac{2}{h} y_{4}(t)\right)+\frac{2}{h}\left(\Delta_{h} y_{4}(t)+\frac{2}{h} y_{4}(t)\right)-f(t) \\
& =\Delta_{h}\left(-\varepsilon t(-1)^{\frac{t}{h}}-\frac{h \varepsilon}{2}(-1)^{\frac{t}{h}}\right)+\frac{2}{h}\left(-\varepsilon t(-1)^{\frac{t}{h}}-\frac{h \varepsilon}{2}(-1)^{\frac{t}{h}}\right) \\
& =-\varepsilon\left\{-\frac{2}{h} t(-1)^{\frac{t}{h}}-(-1)^{\frac{t}{h}}\right\}-\frac{h \varepsilon}{2} \frac{2(-1)^{\frac{t}{h}}}{h}+\frac{2}{h}\left(-\varepsilon t(-1)^{\frac{t}{h}}-\frac{h \varepsilon(-1)^{\frac{t}{h}}}{2}\right) \\
& =\varepsilon(-1)^{\frac{t}{h}}
\end{aligned}
$$

for all $t \in h \mathbb{Z}$. This implies that

$$
\left|\Delta_{h}^{2} y_{4}(t)+\frac{4}{h} \Delta_{h} y_{4}(t)+\frac{4}{h^{2}} y_{4}(t)-f(t)\right|=\varepsilon
$$

holds on $h \mathbb{Z}$. It is easy to check that

$$
x_{4}(t)=c_{1}(-1)^{\frac{t}{h}}+c_{2} t(-1)^{\frac{t}{h}}+Y(t ;-2 / h,-2 / h)
$$

is any solution of (1.1) with $\alpha=4 / h$ and $\beta=4 / h^{2}$, where $c_{1}$ and $c_{2}$ are arbitrary constants. Hence, we obtain $\lim _{t \rightarrow \infty}\left|y_{4}(t)-x_{4}(t)\right|=\infty$ for any $c_{1}$ and $c_{2}$. This completes the proof of Theorem 3.1.

Theorems 1.3 and 3.1 imply the following result immediately.

Theorem 3.2. Let $\lambda_{1}$ and $\lambda_{2}$ be the complex roots of (1.2) with $\lambda_{i} \neq-1 / h$ for $i \in\{1,2\}$. Then (1.1) has Hyers-Ulam stability on $h \mathbb{Z}$ if and only if $\lambda_{i} \neq 0$ and $-2 / h$ for $i \in\{1,2\}$.

\section{CRITERION BY COEFFICIENTS}

In this section, we now focus on the Theorem 3.1 from another angle. Especially, we will establish an instability theorem that can be judged by coefficients. First, we give a lemma.

Lemma 4.1. Let $\lambda_{1}$ and $\lambda_{2}$ satisfy $\left(\lambda-\lambda_{1}\right)\left(\lambda-\lambda_{2}\right)=\lambda^{2}+\alpha \lambda+\beta=0$ with $\lambda_{i} \neq-1 / h$ for $i \in\{1,2\}$. Then there exists $j \in\{1,2\}$ such that $\lambda_{j}=0$ or $\lambda_{j}=-2 / h$ if and only if $\beta=0$ or $\beta=2 \alpha / h-4 / h^{2}$. 
Proof. From the assumption, we see that

$$
\left(\lambda-\lambda_{1}\right)\left(\lambda-\lambda_{2}\right)=\lambda^{2}-\left(\lambda_{1}+\lambda_{2}\right) \lambda+\lambda_{1} \lambda_{2}=0,
$$

and so that $\alpha=-\left(\lambda_{1}+\lambda_{2}\right)$ and $\beta=\lambda_{1} \lambda_{2}$. This says that there exists $j \in\{1,2\}$ such that $\lambda_{j}=0$ if and only if $\beta=0$. Next we will prove that there exists $j \in\{1,2\}$ such that $\lambda_{j}=-2 / h$ if and only if $\beta=2 \alpha / h-4 / h^{2}$ holds. If $\lambda_{1}=-2 / h$ then $\alpha=-\lambda_{2}+2 / h$ and $\beta=-2 \lambda_{2} / h$. Thus, we have

$$
\beta=\frac{-2(-\alpha+2 / h)}{h}=\frac{2 \alpha}{h}-\frac{4}{h^{2}} .
$$

Conversely, this equation implies

$$
\alpha=\frac{h \beta}{2}+\frac{2}{h},
$$

and therefore, we have

$$
\lambda^{2}+\left(\frac{h \beta}{2}+\frac{2}{h}\right) \lambda+\beta=\left(\lambda+\frac{2}{h}\right) \lambda+\frac{h \beta}{2}\left(\lambda+\frac{2}{h}\right)=\left(\lambda+\frac{h \beta}{2}\right)\left(\lambda+\frac{2}{h}\right) .
$$

This says that (1.2) has the root $\lambda=-2 / h$. This completes the proof.

Using Theorem 3.1 and Lemma 4.1, we get the following result.

Theorem 4.1. Suppose that $1-\alpha h+\beta h^{2} \neq 0$. If $\beta\left(\beta-2 \alpha / h+4 / h^{2}\right)=0$, then the equation (1.1) does not have Hyers-Ulam stability on $h \mathbb{Z}$.

Theorems 1.3 and 4.1 imply the following result immediately.

Theorem 4.2. Suppose that $1-\alpha h+\beta h^{2} \neq 0$. Then the equation (1.1) has Hyers-Ulam stability on $h \mathbb{Z}$ if and only if $\beta\left(\beta-2 \alpha / h+4 / h^{2}\right) \neq 0$.

\section{CONCLUSiOnS}

This paper focused on the instability of the second-order nonhomogeneous linear difference equations. It has been shown that Hyers-Ulam stability is broken in the special case where the two coefficients of the equation satisfy $\beta\left(\beta-2 \alpha / h+4 / h^{2}\right)=0$. In the end, we have obtained a necessary and sufficient condition for Hyers-Ulam stability.

Acknowledgements. The author was supported by JSPS KAKENHI Grant Number JP20K03668. The author would like to thank the referee for his/her careful reading and comments.

\section{REFERENCES}

[1] Alsina, C. ; Ger, R. On some inequalities and stability results related to the exponential function. J. Inequal. Appl. 2 (1998), no. 4, 373-380.

[2] Anderson, D. R.; Onitsuka, M. Hyers-Ulam stability of first-order homogeneous linear dynamic equations on time scales. Demonstr. Math. 51 (2018), no. 1, 198-210.

[3] Anderson, D. R.; Onitsuka, M. Hyers-Ulam stability for a discrete time scale with two step sizes. Appl. Math. Comput. 344/345 (2019), 128-140.

[4] Anderson, D. R.; Onitsuka, M. Best constant for Hyers-Ulam stability of second-order $h$-difference equations with constant coefficients Results Math. 74 (2019), no. 4, Art. 151, 16 pp.

[5] Anderson, D. R.; Onitsuka, M. Best constant for Hyers-Ulam stability of two step sizes linear difference equations. J. Math. Anal. Appl. 496 (2021) 124807, 17pp.

[6] Baias, A. R.; Blaga, F; Popa, D. Best Ulam constant for a linear difference equation. Carpathian J. Math. 35 (2019), no. 1, 13-22.

[7] Baias, A. R.; Popa, D. On the best Ulam constant of the second order linear differential operator. Rev. $R$. Acad. Cienc. Exactas Fís. Nat. Ser. A Mat. RACSAM, 114 (2020), no. 1, Paper No. 23, 15 pp.

[8] Baias, A. R.; Popa, D. On the best Ulam constant of a higher order linear difference equation. Bull. Sci. Math. 166 (2021), 102928, 12 pp. 
[9] Brzdęk, J.; Ciepliński, K.; Leśniak, Z. On Ulam's type stability of the linear equation and related issues. Discrete Dyn. Nat. Soc. 2014, Art. ID 536791, 14 pp.

[10] Brzdęk, J.; Popa, D; Raşa, I.; Xu, B. Ulam stability of operators. Mathematical analysis and its applications. Academic Press, 2018.

[11] Jung, S.-M. Hyers-Ulam-Rassias stability of functional equations in nonlinear analysis. Springer Optimization and Its Applications 48, Springer, New York, 2011.

[12] Miura, T.; Miyajima, S.; Takahasi, S.-E. A characterization of Hyers-Ulam stability of first order linear differential operators. J. Math. Anal. Appl. 286 (2003), no. 1, 136-146.

[13] Moslehian, M. S.; Popa, D. On the stability of the first-order linear recurrence in topological vector spaces, Nonlinear Anal. 73 (2010), no. 9, 2792-2799.

[14] Onitsuka, M. Influence of the stepsize on Hyers-Ulam stability of first-order homogeneous linear difference equations. Int. J. Difference Equ.12 (2017), no. 2, 281-302.

[15] Onitsuka, M. Hyers-Ulam stability of first-order nonhomogeneous linear difference equations with a constant stepsize. Appl. Math. Comput. 330 (2018), 143-151.

[16] Onitsuka, M. Hyers-Ulam stability of second-order nonhomogeneous linear difference equations with a constant stepsize. J. Comput. Anal. Appl. 28 (2020), no. 1, 152-165.

[17] Popa, D. Hyers-Ulam stability of the linear recurrence with constant coefficients. Adv. Difference Equ. 2005, no. 2, 101-107.

[18] Popa, D. Hyers-Ulam-Rassias stability of a linear recurrence. J. Math. Anal. Appl. 309 (2005), no. 2, 591-597.

[19] Popa, D.; Raşa, I. On the Hyers-Ulam stability of the linear differential equation. J. Math. Anal. Appl. 381 (2011), no. 2, 530-537.

[20] Takahasi, S.-E.; Miura, T; Miyajima, S. On the Hyers-Ulam stability of the Banach space-valued differential equation $y^{\prime}=\lambda y$. Bull. Korean Math. Soc. 39 (2002), no. 2, 309-315.

[21] Takahasi, S.-E.; Takagi,H.; Miura, T; Miyajima, S. The Hyers-Ulam stability constants of first order linear differential operators. J. Math. Anal. Appl.296 (2004), no. 2, 403-409.

[22] Ulam, S. M. A collection of mathematical problems. Interscience Tracts in Pure and Applied Mathematics no. 8 Interscience Publishers, New York-London, 1960.

[23] Wang, G.; Zhou, M.; Sun, L. Hyers-Ulam stability of linear differential equations of first order. Appl. Math. Lett. 21 (2008), no. 10, 1024-1028.

Department of Applied MATHEMAtics

OKAYAMA UNIVERSITY OF SCIENCE

OKAYAMA 700-0005, JAPAN

Email address: onitsuka@xmath.ous.ac.jp 